\title{
The Sixth Annual International Conference on Urban Health, October 31-November 2, 2006
}

\author{
M. Chris Gibbons and Mary Leach
}

This special section for the Journal of Urban Health presents the submitted abstracts that were awarded oral or poster presentation at the Sixth International Conference on Urban Health in Baltimore, MD. All abstracts were reviewed by the Scientific Advisory Committee.

The theme of the Sixth ICUH was "Harnessing the Power of Technology to Achieve Behavior Change and Improve Urban Health.” This included a focus on the consequences of technology on behavioral interventions and health disparities; knowledge management in urban health; and emerging themes in technology and urban health.

This supplement first presents the submitted abstracts selected for oral presentation (Section I) and then presents submitted abstracts selected for poster presentation (Section II).

The abstracts are arranged according to subject matter, and the corresponding headings are listed below. For the oral sessions, each of the 18 subjects is assigned a number (from 01 to 18 ), the first two numbers of a given abstract. For example, to find Abstract 13-05, the first two digits (13) represent the Cultural and Ethnic Diversity in Urban Health subject, and the last two digits (05) represent the abstract number itself, listed chronologically within each section. For the poster sessions, each of the 18 subjects is assigned a number, preceded by the letter "P" (from P1 to P18), followed by the last two digits that represent the abstract number itself.

\begin{tabular}{ll}
\hline Section I: Oral Sessions & \\
Adolescent and Child Health & $01-01$ to $01-17$ \\
Best Practices in Meeting Urban Health Challenges & $02-01$ to $02-11$ \\
Public Health Informatics in Cities & $03-01$ to $03-12$ \\
Neighborhood Level Influences on Health & $04-01$ to $04-29$ \\
Social Determinants of Health & $05-01$ to $05-13$ \\
Cities and Infectious Diseases & $06-01$ to $06-09$ \\
Environmental Health & $07-01$ to $07-07$ \\
Health Information Technology and Behavior Change & $08-01$ to $08-20$ \\
Community Approaches to Urban Health & $09-01$ to $09-18$ \\
Immigrant and Refugee Populations & $10-01$ to $10-10$ \\
Substance Abuse and Mental Health & $11-01$ to $11-14$ \\
Urban Health from a Global Perspective & $12-01$ to $12-05$ \\
Cultural and Ethnic Diversity in Urban Health & $13-01$ to $13-05$ \\
Other & $14-01$ to $14-02$ \\
Urban Disasters & $15-01$ to $15-05$ \\
Urban Health and GIS & $16-01$ to $16-03$ \\
Demographics: Aging/Gender/Ethnicity & $17-01$ to $17-05$ \\
Urban Health Policy & $18-01$ to $18-03$
\end{tabular}


Section II: Poster Sessions

Adolescent and Child Health

P1-01 to P1-08

Best Practices in Meeting Urban Health Challenges

$\mathrm{P} 2-01$ to $\mathrm{P} 2-03$

Neighborhood Level Influences on Health

P3-01 to 03-06

Social Determinants of Health

P4-01 to $\mathrm{P} 4-10$

Cities and Infectious Diseases

P5-01 to P5-05

Environmental Health

P6-01 to P6-03

Health Information Technology and Behavior Change

P7-01 to P7-02

Community Approaches to Urban Health

P8-01 to P8-05

Immigrant and Refugee Populations

P9-01

Substance Abuse and Mental Health

P10-01 to P10-05

Cultural and Ethnic Diversity in Urban Health

P11-01

Other

$\mathrm{P} 12-01$ to $\mathrm{P} 12-04$

Urban Disasters

P13-01

Urban Health and GIS

P14-01

Demographics: Aging/Gender/Ethnicity

P15-01 to P15-04

Community Partnerships

$\mathrm{P} 16-01$ to $\mathrm{P} 16-04$

Innovation and Entrepreneurship

$\mathrm{P} 17-01$ to $\mathrm{P} 17-02$

Medical Informatics in Cities

P18-01 to P18-02

\section{Abstracts Supplement Disclaimer}

The opinions or views expressed in this supplement are those of the authors and do not necessarily reflect the opinions or recommendations of the conference organizers, sponsoring agencies, the journal, or the publisher. The abstracts have not undergone review by the Editorial Board of the Journal of Urban Health. The selection of abstracts has been the responsibility of the Scientific Advisory Committee of the Sixth International Conference on Urban Health. The publisher has endeavored to reproduce faithfully all the abstracts as accepted. 